
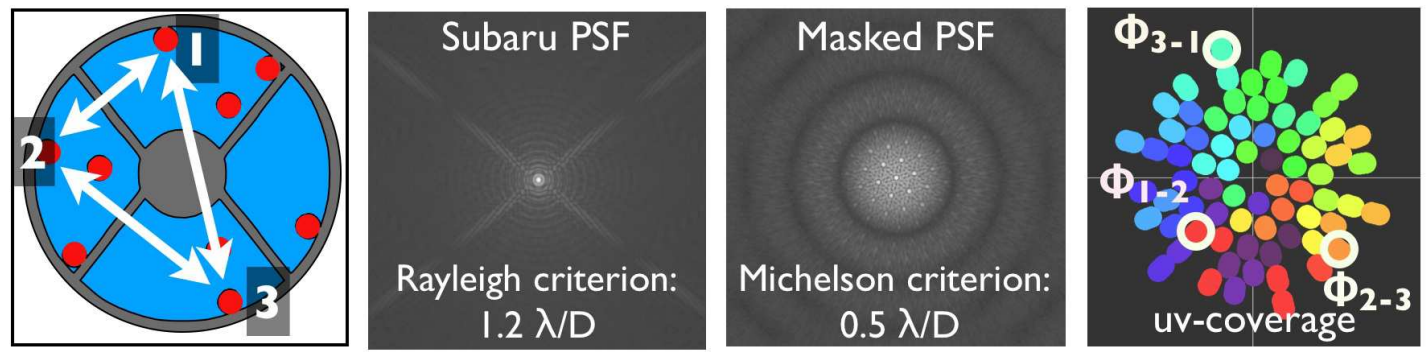

FIGURE 1. Example of non-redundant aperture mask that accomodates the Subaru telescope pupil.

\title{
Aperture Masking Interferometry for Subaru
}

\author{
Frantz Martinache, Olivier Guyon and Vincent Garrel
}

\author{
Subaru Telescope
}

\begin{abstract}
Aperture Masking Interferometry used in combination with Adaptive Optics, is a powerful technique that permits the detection of faint companions at small angular separations. The precision calibration of the data achieved with this observing mode indeed leads to reliable results up to and beyond the formal diffraction limit, explaining why it has, in just a few years, been ported on most major telescopes. In this poster, we present its possible implementation on Subaru. We also discuss how the opportunity offered by the planned Extreme-AO upgrade to HiCIAO will push further the performance of this already successful technique, offering Subaru a unique access to a very exciting region of the "contrast-ratio - angular separation" parameter space.
\end{abstract}

Keywords: Technique:Interferometry, Adaptive Optics

Initially developed for radio astronomy [2], non-redundant baseline interferometry is now used in IR and optical bandpasses [1, 6]. A modern description of NRM can be found in Tuthill et al. [7], Monnier [4] and references therein. In brief, an N-hole pupil mask turns the extremely redundant full aperture of a telescope into a simpler interferometric array. Taking into account the geometry of the pupil (segmentation, central obscuration and spider vanes) and the bandwidth, the mask is designed such that each baseline (a vector linking the centers of two holes) is unique: the mask is said non-redundant in the sense that each spatial frequency is only sampled once (Fig. 1).

In a sense, NRM is a form of imaging with an unsual-looking PSF that displays multiple sharp peaks, whereas a traditional diffraction-limited PSFs is dominated by one only peak. The real virtues of the NRM PSF however appear once the image is Fourier transformed (e.g. Fig. 1 right panel) as all spatial frequencies sampled by the mask appear as well separated peaks containing both amplitude and phase information. 


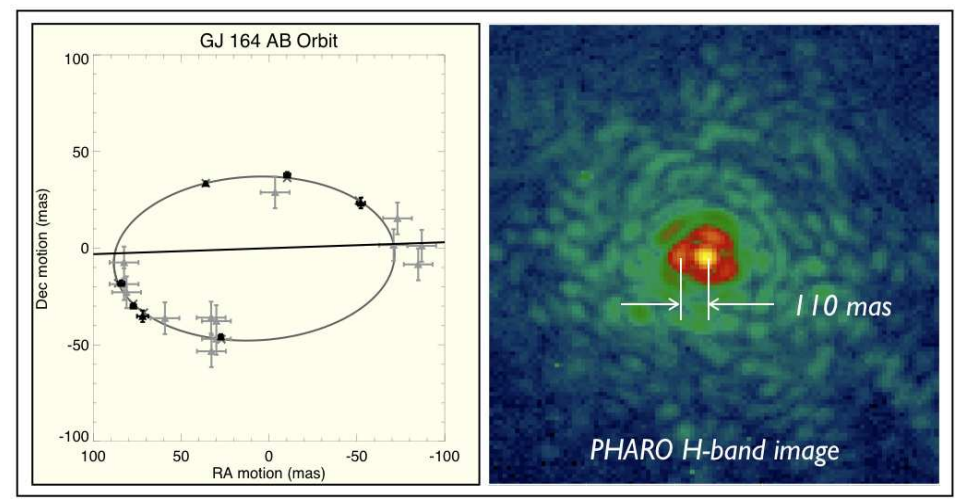

FIGURE 2. Example of result obtained with the proposed technique with the instrument PHARO at the Cassegrain focus of the Palomar Hale telescope. The 5-meter aperture was able to resolve (black points) the M8 companion orbiting the star GJ 164 at separations between ranging from 40 to 80 mas, in $\mathrm{H}$ - and K-bands [3].

The advantages of this approach are that:

- The longest baseline provides a resolving power of $0.5 \lambda / D$ to be compared the traditionally accepted limit of $1.22 \lambda / D$ known as the Rayleigh criterion.

- Unlike conventional PSF co-addition, the information extracted from the NRM data can be averaged to reduce noise, even in the presence of slowly-varying speckles.

- Non-redundancy ensures that the complex visibilities can be used to form closure phases [1], an observable that calibrates wavefront residual errors as well as noncommon path errors between the science and sensing arms of the instrument.

- The outer working angle (OWA) is set by the shortest baseline, typically $4 \lambda / D$ : the NRM search space nicely complements that of coronagraphy.

It may seem perverse to throw away most of a telescope's collecting area, but the $10-20 \%$ mask transmission price paid for NRM (comparable to most high performance coronagraphs' throughput) purchases not only a straightforward $2.44 \times$ gain in resolution but also a dramatic increase in signal to noise ratio. Today's ground-based NRM routinely achieves stability of 0.5 degrees on the closure phase, hence passively stabilizing the phase at the level of $\lambda / 500$ to $\lambda / 1000$, a performance that will only be matched by the next generation of ExAO coronagraphic systems [5].

\section{REFERENCES}

1. Baldwin, J. E., Haniff, C. A., Mackay, C. D., \& Warner, P. J. 1986, Nature, 320, 595

2. Jennison, R. C. 1958, MNRAS, 118, 276

3. Martinache, F., Rojas-Ayala, B., Ireland, M. J., Lloyd, J. P., \& Tuthill, P. G. 2009, ApJ, 695, 1183

4. Monnier, J. D. 2003, Reports of Progress in Physics, 66, 789

5. Sivaramakrishnan, A., Soummer, R., Pueyo, L., Wallace, J. K., \& Shao, M. 2008, ApJ, 688, 701

6. Tuthill, P. G., Monnier, J. D., Danchi, W. C., \& Haniff, C. A. 1998, in Proc. SPIE, ed. R. D. Reasenberg, Vol. 3350, 839-846

7. Tuthill, P. G., Monnier, J. D., Danchi, W. C., Wishnow, E. H., \& Haniff, C. A. 2000, PASP, 112, 555 